Chapter 10

\title{
Kinetics of Growing Centimeter Long Carbon Nanotube Arrays
}

\author{
Wondong Cho, Mark Schulz and Vesselin Shanov \\ Additional information is available at the end of the chapter \\ http://dx.doi.org/10.5772/50837
}

\section{Introduction}

Carbon nanotubes (CNTs) are fascinating materials with outstanding mechanical, optical, thermal, and electrical properties [1-4]. CNTs also have a huge aspect ratio and a large surface area to volume ratio. Because of their unique properties, vertically aligned centimeter long CNT arrays have generated great interest for environmental sensors, biosensors, spinning CNT into yarn, super-capacitors, and super-hydrophobic materials for self-cleaning surfaces [5-11]. Yun et al. studied a needle-type biosensor based on CNTs to detect dopamine. Their results showed advantages of using CNT biosensors for detecting neurotransmitters [11]. Most of the envisioned applications require CNTs with high quality, a long length, and well aligned vertical orientation. Although many researchers have studied the synthesis of vertically aligned CNT arrays, the CNT growth mechanism still needs to be better understood. In addition, CNT lengths are typically limited to a few millimeters because the catalyst lifetime is usually less than one hour [12-16]. Many groups have studied the kinetics of CNT growth trying to improve CNT properties. Different observation methods [17-22] were used to determine the effect of the catalyst, buffer layers, carbon precursor, and deposition conditions on nanotube growth. One of the suggested growth mechanisms postulates several steps [23]. First, the carbon source dissociates on the surface of the substrate. Next, the carbon atoms diffuse to the molten catalyst islands and dissolve. The metal-carbon solution formed reaches a supersaturated state. Finally, the carbon nanotubes start to grow from the carbon- catalyst solution. In situ observation of CNTs during their nucleation and growth is a useful method to understand the growth mechanism, which might help to overcome the limitation of the short length of nanotubes, and to control array growth and quality. Various remarkable approaches of in situ observation have been performed to affirm the growth mechanism of vertically aligned CNTs and also to obtain kinetics data such as 
growth rate and activation energy [12, 24-27]. Puretzky et al. studied the growth kinetics of CNT arrays using in situ time-lapse photography and laser irradiation under diffusion-limited growth conditions [28]. In situ transmission electron microscopy (TEM) was used by Kim et al. to study the dynamic growth behavior of CNT arrays [29]. Additionally, a pseudo in situ monitoring method was used to investigate the kinetics of CNT array growth by creating marks on the side of the CNT array during the growth. Using this method, several groups demonstrated root growth for their catalyst systems. However, their studies were limited to short lengths and also required ex-situ observation with SEM to obtain the growth length as a function of time $[25,27,30,31]$. Most reported methods are designed to operate and monitor the growth length with time for relatively short (a few millimeters long) CNT arrays and also do not provide kinetics data for growing centimeter long CNT arrays.

Recently, we have developed new catalyst systems which are able to grow over 1 centimeter long vertically aligned CNT arrays [6]. In this paper we examined the growth mechanism and kinetics of centimeter long carbon nanotube arrays using a real-time photography technique and the effect of growth temperature and growth time on morphology of CNTs.

\section{Experimental Method}

Fig. 1 shows the schematic of the chemical vapor deposition (CVD) system used for centimeter long CNT array growth. The reactor consists of a 2 inch quartz tube placed inside a high temperature furnace (Barnstead International, type F79400 tube furnace) and four massflow controllers (MFC) which control the flow rate of the gas reactants such as hydrogen, ethylene, water vapor and argon. A water bubbler is also installed to provide water vapor using argon carrier gas. A window on the side of the reactor is used to acquire real-time images of CNT arrays and to record data with a digital camera (Olympus E510) controlled by a computer.

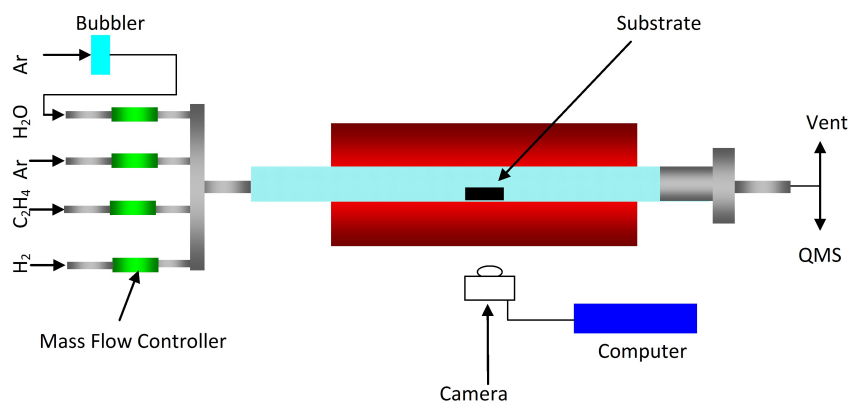

Figure 1. Schematic of the CVD system for direct observation of the centimeter long carbon nanotube arrays during their growth. The top view of the reactor is shown.

The substrates were parts of 4 inch silicon wafers (100) with $\mathrm{SiO}_{2}(500 \mathrm{~nm})$ on the top. The buffer and catalyst layers based on $\mathrm{Al}_{2} \mathrm{O}_{3}(15 \mathrm{~nm}$ thick $) / \mathrm{Fe}(1 \mathrm{~nm}$ thick) were deposited on the 
wafers using e-beam evaporation. After the deposition, the substrates were annealed for several hours at $400{ }^{\circ} \mathrm{C}$ in Air. All the experiments were performed using the following optimized recipe for centimeter long CNT arrays: $560 \mathrm{mmHg}$ of argon, $60 \mathrm{mmHg}$ of hydrogen, and $140 \mathrm{mmHg}$ of ethylene as a carbon precursor. The water concentration in the reactor was near $900 \mathrm{ppm}$ measured by a quadrupole mass spectrometer (QMS). The total pressure was kept at one atmosphere during the growth and the temperature varied from $690{ }^{\circ} \mathrm{C}$ to $840{ }^{\circ} \mathrm{C}$. Real-time images of the $\mathrm{CNT}$ array growth were recorded from the moment that ethylene was introduced into the reactor. The images were used to study the growth mechanism and kinetics of the CNT growth. Scanning electron microscopy (SEM, Phillips XL30 ESEM), high resolution transmission electron microscopy (HRTEM, JOEL 2000 FX) and MicroRaman spectroscopy (Renishaw inVia Reflex Micro-Raman) were employed to characterize the CNT morphology.

\section{Results and discussion}

\subsection{Growth evolution by real-time photography}

Real-time photography was used to study the growth mechanism and kinetics of centimeter long CNT array growth. The digital camera provided clear images showing details related to the dynamic changes of the array shape during the growth. This was achieved by controlling the intervals for taking pictures from a few seconds to several hours depending on the experimental conditions.

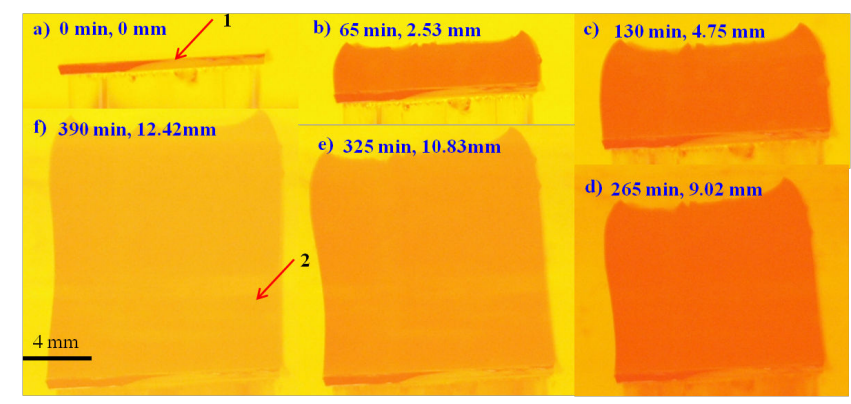

Figure 2. Real-time images of the centimeter long CNT array growth evolution with time during CVD at $780^{\circ} \mathrm{C}:(\mathrm{a})$ Side image of the substrate at zero growth time, (b) to (f) Images of CNT arrays grown for different times.

Fig. 2 illustrates sequential images of vertically aligned centimeter long CNT arrays grown at $780{ }^{\circ} \mathrm{C}$. As can be seen from Fig. 2, it is easy to distinguish the substrate from the CNT array. Arrow 1 in Fig. 2a points to a side view of the substrate. Arrow 2 in Fig. $2 \mathrm{f}$ shows the side view of the CNT array. The growth length can be obtained as a function of the deposition time from the images. Changes in the array shape can also be observed during the entire growth time. In Fig. 2f, the growth length was $12.47 \mathrm{~mm}$ and the catalyst lifetime was 450 min. This experiment was repeated several times at the same deposition conditions and the results were reproducible. Hence, Fig. 2 demonstrates the real-time images which allow 
measuring the growth length and observing the CNT array growth mechanism. This approach gives reproducible results for studying the kinetics of CNT array growth.

Movies composed of multiple images were created to investigate the morphological changes and growth mechanism of CNT arrays during their synthesis (Supporting material 1).

\subsection{Growth mode of centimeter long CNT arrays}

A carbon source interruption method combined with real-time photography was used to determine the CNT array growth mechanism (root vs. tip growth). Fig. 3 shows real-time images of CNT arrays obtained for different periods of growth employing 5 minute interruption of the ethylene supply. In this experiment, the ethylene flow was first stopped after 70 minutes growth while the rest of reactant gases such as $\mathrm{Ar}, \mathrm{H}_{2}$ and water vapor were continuously supplied with same partial pressure. Next, the ethylene was resupplied after the $5 \mathrm{~min}$ break. Fig. 3b, c and d show real-time images of CNT arrays before and after the 5 minute interruption of the carbon precursor. The growth length in Fig. $3 \mathrm{~b}$ was $2.89 \mathrm{~mm}$. The length did not change after the ethylene interruption (Fig. 3c). CNTs started to grow again after ethylene was resupplied (Fig. 3d). As can be seen in Fig. 3e and f, the first layer which grew before the $5 \mathrm{~min}$ ethylene interruption detached from the second layer grown after the $5 \mathrm{~min}$ interruption. The separation may be caused by water etching the interface between the root of the CNTs and the substrate during the interruption. Even though the first layer detached, the second layer continued to grow from the bottom. Fig. 3f shows that the growth length of the second layer was $2.85 \mathrm{~mm}$. The calculated growth rate was $38.5 \mu \mathrm{m} / \mathrm{min}$ before and after the interruption and until the growth stopped.

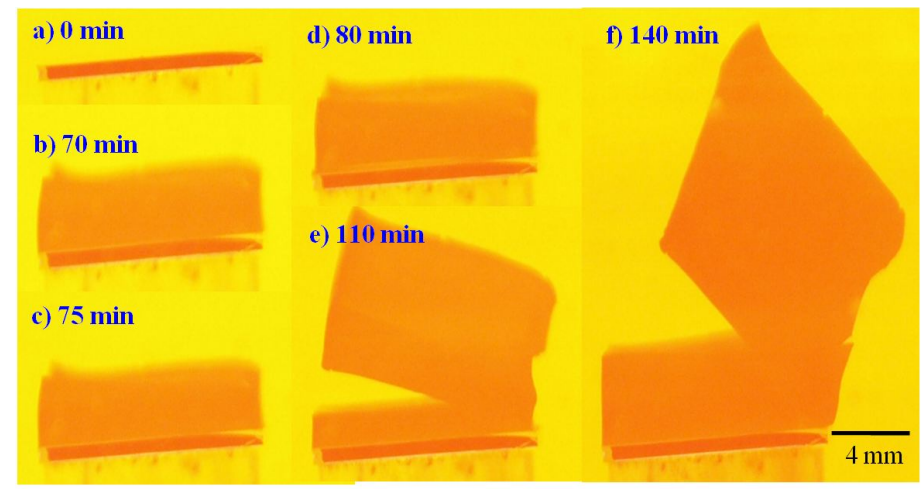

Figure 3. Real-time images of CNT array grown for different periods of time at a deposition temperature of $780{ }^{\circ} \mathrm{C}$ with 5 minutes interruption of ethylene: (a) Side image of the substrate at zero growth time, (b) to (f) Pictures of CNT arrays grown for different times.

It was obvious from the data that the interruption didn't affect the growth rate during the CNT synthesis. The images also reveal that the growth pathway of the centimeter long CNT array is "root growth" (Supporting materials 2). 
The top surface of the centimeter long CNT array was studied by Energy Dispersive X-ray Spectroscopy (EDS) to determine if metal catalyst moved to the tips of the CNTs. No trace of iron catalyst was detected on the top of the CNT array. Thermogravimetric analysis (TGA) performed at a heating rate of $10^{\circ} \mathrm{C} / \mathrm{min}$ in air showed that the change of CNT array weight was negligible at temperatures below $550{ }^{\circ} \mathrm{C}$. The combustion started slightly below $700{ }^{\circ} \mathrm{C}$ and was completed at $750^{\circ} \mathrm{C}$. The amount of residual matter was extremely small and was not measureable after the completion of combustion above $750{ }^{\circ} \mathrm{C}$. These results implied that the CNT array was almost "catalyst free".

\subsection{Kinetics of centimeter long CNT array growth}

The change in the growth length as a function of deposition time at different temperatures was investigated using real-time photography. Fig. 4 displays the dependence of growth length on the growth time in the range of $730{ }^{\circ} \mathrm{C}$ to $840{ }^{\circ} \mathrm{C}$. The rest of the CVD experimental conditions were kept constant. As shown in Fig. 4a, the growth length increases linearly with the growth time within the entire temperature range of $730^{\circ} \mathrm{C}$ to $840{ }^{\circ} \mathrm{C}$. The data show that the growth rate remained constant until the catalyst was deactivated. Diffusion of the carbon precursor through the CNT forest apparently did not limit the growth rate even in the case of $12 \mathrm{~mm}$ long CNT arrays. It reveals the carbon source was able to reach the surface of catalyst particles without significant resistance. These results show that the growth rate followed a kinetic controlled mode. Zhu et al. reported that their catalyst system was controlled by a gas diffusion process and their growth rate decreased gradually with increasing the length of CNT array. They reported a diffusion controlled mode for a similar range of growth temperatures [31] used in the present paper.

In the current experiments, growth termination occurred abruptly for all experimental deposition temperatures. After growth termination, the array length remained constant with time. The reason for such abrupt catalyst deactivation is not clear and it could not be interpreted using the suggested mechanisms in the literature such as Oswald ripening, forming stable iron carbides, and depletion of the catalyst [32-34]. It is hypothesized that catalyst deactivation occurs due to several complex reasons. One reason is that amorphous carbon is built up during the CVD process and covers the catalyst active site at the surface of the substrate which results in passivation of the catalyst.

Fig. $4 \mathrm{~b}$ shows the effect of temperature on the final growth length. The slope of the curve increases gradually as the CVD temperature increases from $730^{\circ} \mathrm{C}$ to $780^{\circ} \mathrm{C}$, and then decreases. The longest $\mathrm{CNT}$ length of $12.42 \mathrm{~mm}$ was achieved at $780^{\circ} \mathrm{C}$. It was observed that the longest centimeter long CNT array was obtained neither at the highest growth rate nor at the longest catalyst lifetime. Analyzing Fig. 4 provides a better understanding of the catalyst lifetime at different temperatures. Fig. 4c shows that the catalyst lifetime decreases linearly with the increase of temperature in the range of $730{ }^{\circ} \mathrm{C}$ to $840{ }^{\circ} \mathrm{C}$. In this experiment, the longest catalyst lifetime was $895 \mathrm{~min}$ at $730^{\circ} \mathrm{C}$. The catalyst lifetime decreases dramatically down to a few minutes as the temperature approaches to $840{ }^{\circ} \mathrm{C}$. The results in this paper demonstrate that the catalyst lifetime and the final growth length are considerably longer than reported in the literature [12-16]. Water vapor usually plays an important role in enhancing the growth rate and also prolonging the catalyst lifetime [35] since water vapor may clean the surface of the catalyst particles by removing the amorphous carbon. 

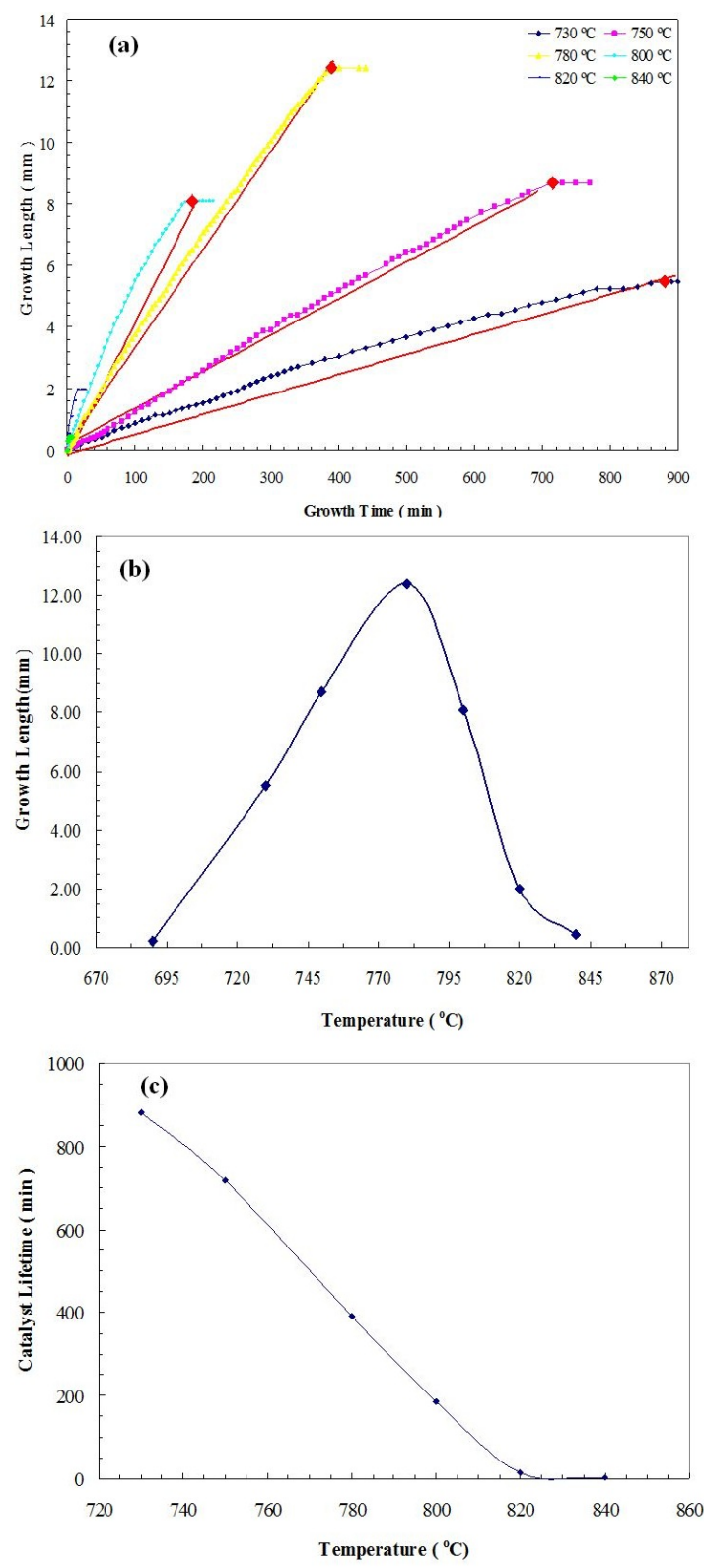

Figure 4. Experimental results from the kinetics study of centimeter long CNT array growth: (a) Growth length as a function of the growth time. The solid red lines indicate the fitting of the analytical growth equation to the experimental results. (b) Plot of the final growth length after termination vs. deposition temperature. (c) Plot of the catalyst lifetime as a function of the temperature. 
Fig. 5 shows the Arrhenius plot of the CNT growth rate as a function of the temperature. The growth rate was calculated using the final growth length divided by the catalyst lifetime. The highest growth rate achieved was $193 \mu \mathrm{m} / \mathrm{min}$ at $840{ }^{\circ} \mathrm{C}$. The present results reveal that the growth rate is proportional to the concentration of ethylene, so first order reaction is assumed. The activation energy $E_{a}$ was calculated based on the Arrhenius equation $k=k_{0}$ $\exp \left(-E_{a} / R T\right)$ where, $\mathrm{k}_{0}$ is the frequency factor, $\mathrm{T}$ is the growth temperature $(\mathrm{K}), k$ is the reaction rate constant (in this case $k$ is growth rate constant), and $\mathrm{R}$ is the ideal gas constant. The activation energy calculated from Arrhenius equation and Fig. 5 was about $248 \mathrm{~kJ} / \mathrm{mol}$. Similarly, Zhu et al. obtained activation energy of $201.2 \mathrm{~kJ} / \mathrm{mol}$ for a $\mathrm{Fe} / \mathrm{Al}_{2} \mathrm{O}_{3} / \mathrm{Si}$ substrate using ethylene as a carbon source [27]. Li et al also reported activation energy of $158 \mathrm{~kJ} / \mathrm{mol}$ for the temperature interval of $730{ }^{\circ} \mathrm{C}$ to $780{ }^{\circ} \mathrm{C}$ using ethylene [24]. Both groups concluded that the growth rate was not affected by the diffusion of the carbon source to the catalysts because the CNT length increased linearly with time. In the current paper, the same trend is observed although the catalyst lifetime and CNT growth length were much longer.

Based on the kinetics data, it was concluded that the centimeter long CNT array growth does not fit the diffusion controlled mode and is more reasonable to be considered as a kinetically controlled process. This conclusion is based on the specific process conditions and size of the substrate used here. Diffusion may play a role in growth for other experimental conditions.

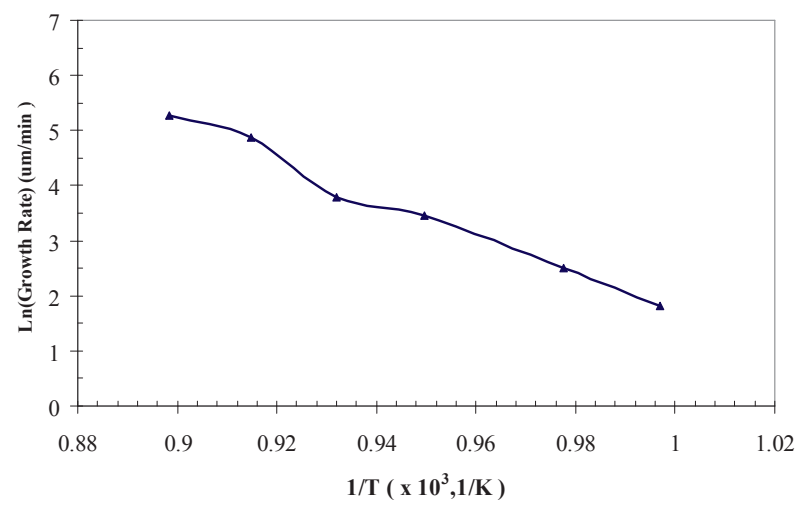

Figure 5. Arrhenius plot of centimeter long CNT array growth rate as a function of $1 /$ temperature.

Zhu et al. adopted the silicon oxidation model for a diffusion controlled mode of CNT growth described with the equation: $h_{0}=0.5\left(A^{2}+4 B t\right)^{1 / 2}-0.5 A$. Futaba et al. assumed that the deactivation of catalyst was analogous to radioactive decay to model their super growth of CNT via the equation: $H(t)=\beta \tau_{0}\left(1-e^{-t / \tau 0}\right)$. They demonstrated a good fit of the experimental data to the model equations. However, Futaba et al. could not extrapolate it for a longer growth time in order to obtain the final growth length and to predict the abrupt growth termination. Hence, the models described above cannot be adopted for the centimeter long CNT array growth. Based on the experimental data in this paper, it was attempted to derive 
a growth model using an analytical method that can be reasonably applied to the growth of centimeter long CNT arrays.

From Fig. 4, the growth length can be expressed by a linear equation:

$$
G L_{T}(t)=G L_{T f}-r_{g}\left(t_{T f}-t\right)
$$

where, $G L_{T}(t)$ is the array length at a certain growth time $t$ and temperature $T, G L_{T f}$ is the final growth length of the centimeter long CNT array after termination at temperature $T, r_{8}$ is the growth rate and $t_{T f}$ is the catalyst lifetime. The final growth length $G L_{T f}$ can be obtained by the following equation depending on the deposition temperature:

In case of temperatures below $780{ }^{\circ} \mathrm{C}$, the final CNT length

$$
G L_{T f}=0.1367 T(\stackrel{\circ}{ } C)-94.1
$$

Above deposition temperatures of $780^{\circ} \mathrm{C}$, the final $\mathrm{CNT}$ length is

$$
G L_{T f}=-0.216 T(\stackrel{\circ}{ } C)+181
$$

The catalyst lifetime $t_{T f}$ can also be expressed by a linear equation from Fig. 6b:

$$
t_{T f}=-10.08 T(\stackrel{\circ}{ } \mathrm{C})+8253.7 \text {. }
$$

The solid red lines displayed in Fig. 4a are plotted based on the equation (1). As shown in Fig. $4 \mathrm{a}$, the plot fits the experimental data well. The analytical model can also predict the final growth length, growth rate, and catalyst lifetime for a certain CVD temperature.

\subsection{Morphology of centimeter long CNTs}

Fig. 6a, b and c display SEM images of centimeter long CNT arrays obtained at different magnifications. At low magnification of 1000x (Fig. 6a) the image shows appearance of vertically aligned CNTs. Fig. $6 \mathrm{~b}$ and $\mathrm{c}$ are taken at higher magnifications and reveal individual CNTs. The images indicate that despite the long growth time and centimeter length, the tubes grew vertically without any interruption until the catalyst activity was terminated.

HRTEM was used to study changes in the structure and diameter distribution of individual CNTs at different growth temperatures. The images shown in Fig. 7 reveal well defined multi-wall CNTs without metal catalyst incorporated into the tubes. At low temperatures amorphous carbon deposited on the walls was also observed.

Fig. 7e and $\mathrm{f}$ show the distribution of number of walls and average tube diameter of each wall at different growth temperatures. The diameter of CNTs is in the range of 5 to $9 \mathrm{~nm}$ and is independent on the number of walls. 


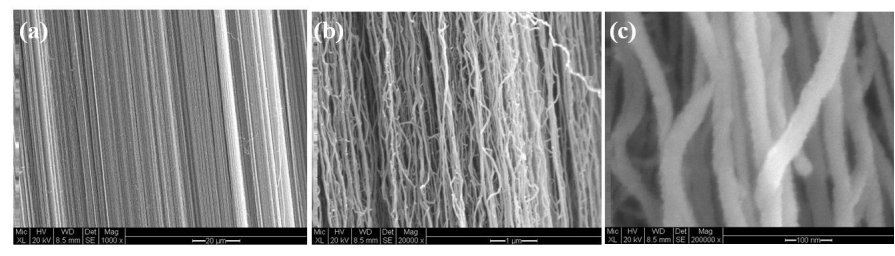

Figure 6. SEM images showing side view of a centimeter long CNT array at different magnifications. The magnification increases from (a) to (b) and (c).

This trend was observed to be similar for every growth temperature used. We found that the diameter distribution does not depend on the growth temperature. The distribution of the number of walls also revealed broad range from 1 to 10 walls for each growth temperature in Fig. 7e. Most of the tubes possess 2 to 5 walls at each growth temperature. We observed that the single wall CNTs were produced at $800{ }^{\circ} \mathrm{C}$ and the double wall CNTs were yielded above $750{ }^{\circ} \mathrm{C}$. In our experiments, the distribution of the walls was not substantially affected by the growth temperature. Hence, we noted that the growth temperature is not an important factor affecting the structure of CNTs obtained in our growth process.
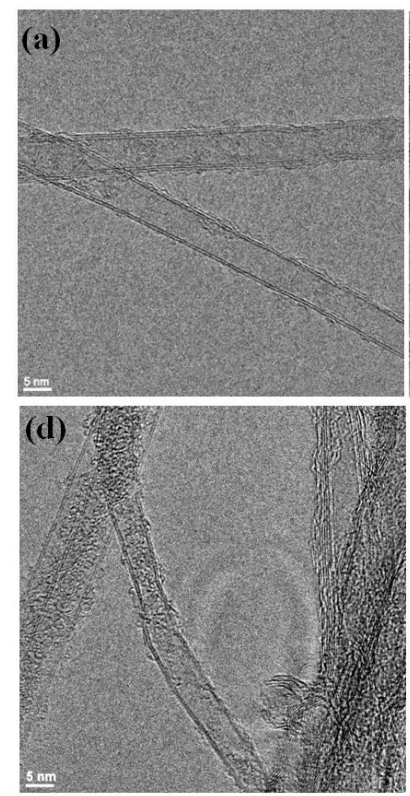
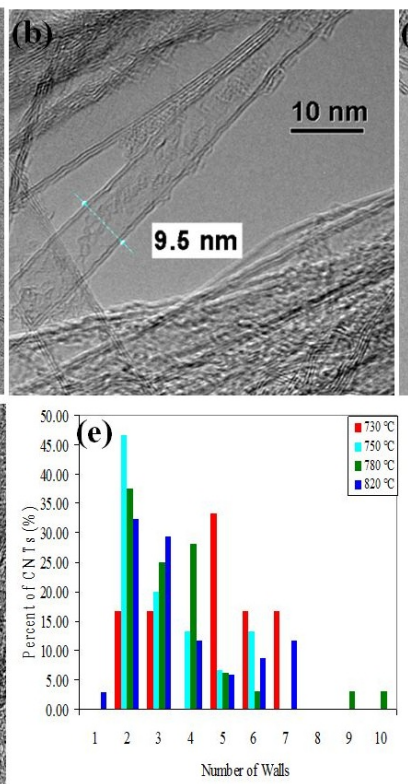
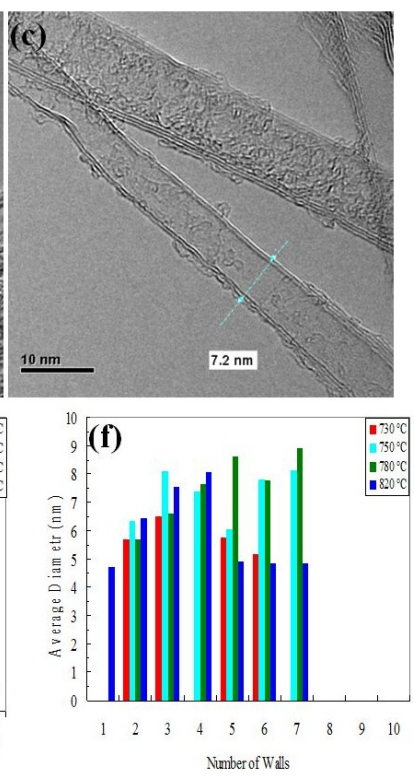

Figure 7. HRTEM images and distribution of the CNTs walls at different growth temperatures: (a) $730^{\circ} \mathrm{C}$, (b) $7500^{\circ} \mathrm{C}$, (c) $780^{\circ} \mathrm{C},(d) 820^{\circ} \mathrm{C}$, (e) distribution of number of walls, (f) distribution of the average tube diameter of each wall for different multiwall CNTs. 

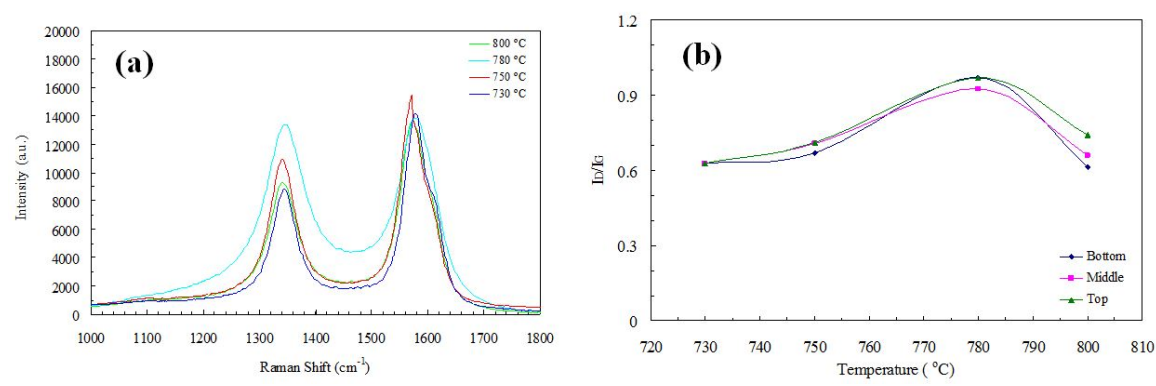

Figure 8. Raman spectra of CNTs taken from the middle position of the CNTs: (a) Spectra at various growth temperatures, (b) $I_{D} / I_{G}$ peak intensity ratios as a function of growth temperature.
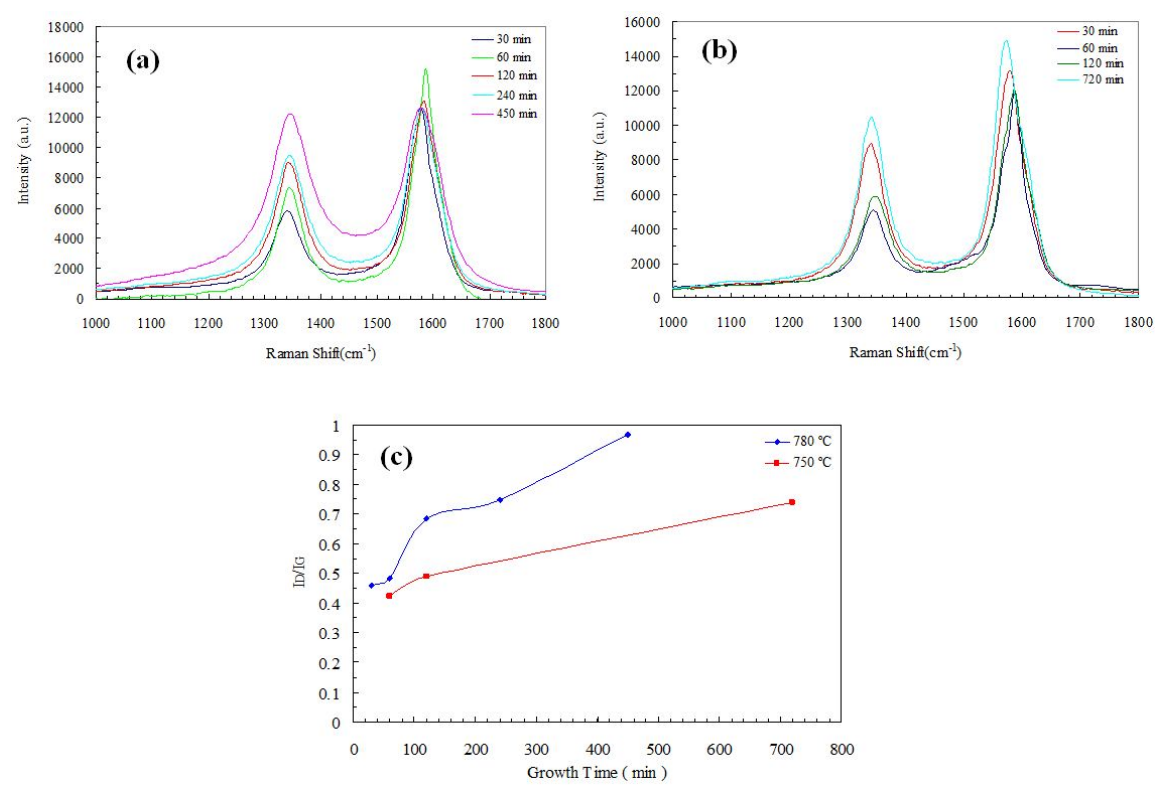

Figure 9. Raman spectra of CNTs synthesized at different temperature and growth time: (a) spectra at $780{ }^{\circ} \mathrm{C}$, (b) spectra at $750^{\circ} \mathrm{C}$, (c) $I_{D} / I_{G}$ peak intensity ratios vs. different growth time.

Micro-Raman spectroscopy (Renishaw inVia Reflex Micro-Raman) was used to investigate the effect of temperature and growth time on the quality of CNT arrays using a $514 \mathrm{~nm}$ excitation wavelength. Fig. 8 shows Raman shift and $\mathrm{I}_{\mathrm{D}} / \mathrm{I}_{\mathrm{G}}$ ratio of the CNTs at different growth temperatures for three points along the array length. The spectra show distinguished D band peak (near $1350 \mathrm{~cm}^{-1}$ ) which indicates presence of defects, disordered and amorphous carbon. A pronounced G band peak (at $1580 \mathrm{~cm}^{-1}$ ) originating from graphitization of CNTs is also displayed [36-40]. As shown in Fig. 8a, the intensity of the D band increased with in- 
creasing of the growth temperature up to $780{ }^{\circ} \mathrm{C}$. Fig. $8 \mathrm{~b}$ displays the intensity ratio $\mathrm{I}_{\mathrm{D}} / \mathrm{I}_{\mathrm{G}}$ as a function of the growth temperature from three different height positions of CNTs. The intensity ratio $\mathrm{I}_{\mathrm{D}} / \mathrm{I}_{\mathrm{G}}$ was similar at bottom, middle and top positions for each growth temperature and rose from 0.62 to 1 as the temperature increases with a maximum at $780{ }^{\circ} \mathrm{C}$. Fig. 9 shows Raman data for CNT arrays obtained at different growth time and temperatures. The intensity of $\mathrm{D}$ peak increases linearly with rising the growth temperature as shown on Fig. $9 \mathrm{a}$ and b. Fig. $9 \mathrm{c}$ illustrates that the $\mathrm{I}_{\mathrm{D}} / \mathrm{I}_{\mathrm{G}}$ ratio also increases near linearly with extending the growth time, which indicates that the quality of CNTs deteriorates when the tubes reside longer in the growth zone. At $750{ }^{\circ} \mathrm{C}, \mathrm{I}_{\mathrm{D}} / \mathrm{I}_{\mathrm{G}}$ increased from 0.422 to 0.704 when prolonging the growth time. The highest value 0.968 of $\mathrm{I}_{\mathrm{D}} / \mathrm{I}_{\mathrm{G}}$ was obtained at $780{ }^{\circ} \mathrm{C}$. Fig. $9 \mathrm{C}$ indicates that $\mathrm{I}_{\mathrm{D}} / \mathrm{I}_{\mathrm{G}}$ values at $780{ }^{\circ} \mathrm{C}$ are greater than those obtained at $750{ }^{\circ} \mathrm{C}$.

As the growth time increases, the gap between the two plots in Fig 9c broadens. Thus, the quality of CNTs decreases faster with time at higher temperatures. The reason for this is the accumulation of the amorphous carbon at high temperatures. These results are supported by the presented HRTEM images.

\section{Conclusions}

Real-time photography was used to record the growth of centimeter long CNT arrays during the CVD process. The kinetics of growing vertically aligned CNTs was studied based on the photographic images. Furthermore, we found that the CNT arrays grew by the root growth mechanism which was proved by the carbon source interruption method and realtime photography. The length of the CNT arrays increased linearly with growth time for all the tested temperatures followed by an abrupt growth termination. The catalyst lifetime decreased linearly with rising the deposition temperature and varied from a few minutes up to several hundred minutes depended on the growth conditions. We found out that the formation of centimeter long CNT array could not be described by diffusion controlled or exponentially decaying growth. This study suggests that the growth in this case is governed by a kinetically controlled mode within the temperature interval from $730{ }^{\circ} \mathrm{C}$ to $840{ }^{\circ} \mathrm{C}$. The calculated activation energy is $248 \mathrm{~kJ} / \mathrm{mol}$. An analytical model for centimeter long CNT array synthesis was proposed which can predict the growth rate, final CNT length, and the catalyst lifetime. The obtained data indicated that the wall and diameter distribution of CNTs is independent on the growth temperature. The quality of CNTs deteriorates with increasing of the growth time and temperature. We found out that the amount of amorphous carbon on the CNTs depends on the residence time of the tubes in the CVD growth zone and on the deposition temperature. Longer residence time and higher deposition temperature accumulates greater amount of amorphous carbon. 


\section{Acknowledgements}

The financial support from NSF through grant CMMI-07272500 and from NCA\&T through DURIP-ONR is highly acknowledged. We also would like to thank Jay Yocis who helped to set up the real-time photography system and Dr. John Robertson from Cambridge University who suggested real time photography of studying kinetics of CNT arrays.

Appendix A. Supplementary data

Movies showing the centimeter long CNT array growth inside of the CVD reactor, including the root growth are available online.

\section{Author details}

Wondong $\mathrm{Cho}^{1,2}$, Mark Schulz ${ }^{2,3}$ and Vesselin Shanov ${ }^{1,2^{*}}$

*Address all correspondence to: vesselin.shanov@uc.edu

1 Chemical and Materials Engineering, University of Cincinnati, Cincinnati, Ohio USA

2 Nanoworld Laboratory, Rh414, University of Cincinnati, Cincinnati, Ohio USA

3 Mechanical Engineering, University of Cincinnati, Cincinnati, Ohio USA

\section{References}

[1] Puretzky, A. A., Geohegan, D. B., \& Eres, G. (2008). Real-time imaging of vertically aligned carbon nanotube array growth kinetics. Nanotechnology, 19, 055605.

[2] Amama, P. B., Pint, C. L., Mc Jilton, L., Kim, S. M., Stach, E. A., Murray, P. T., Hauge, R. H., \& Maruyama, B. (2008). Role of Water in Super Growth of Single-Walled Carbon Nanotube Carpets. Nano Letters, 9, 44-49.

[3] Baughman, R. H., Zakhidov, A., \& de Heer, W. A. (2002). Carbon nanotubes-the route toward applications. Science, 297, 787-792.

[4] Bronikowski, M. (2007). Longer nanotubes at lower temperatres: the influence of effective activation energies on carbon nanotube growth by thermal chemical vapor deposition. J. Phys. Chem. C, 111, 17705-17712.

[5] Gommes, C., Pirard, J. P., \& Blacher, S. (2004). Influence of the operating conditions on the production rate of multi-walled carbon nanotubes in a CVD reactor. Carbon, 42, 1473-1482. 
[6] Vix-Guterl, C., Couzi, M., \& Delhaes, P. (2004). Surface charactrizations of carbon mutiwall nanotubes:comparison surface active sites and raman spectroscopy. J. Phys. Chem. B, 108, 19361-19367.

[7] Kim, D. H., Lee, H. R., \& Jang, H. S. (2003). Dynamic growth rate behavior of a carbon nanotube forest characterized by in situ optical growth monitoring. Nano Letters, 3, 863-865.

[8] Du, C., Yeh, J., \& Pan, N. (2005). High power density supercapacitors using locally aligned carbon nanotube electrodes. Nanotechnology, 16, 350-353.

[9] Einarsson, E., \& Maruyama, Y. M. S. (2008). Growth dynamics of vertically aligned single-walled carbon nanotubes from in situ measurements. Carbon , 46, 923-930.

[10] Mckee, G. S. B., , J. S. F., \& Vecchio, K. S. (2008). Length and the oxidation kinetics of chemical vapor deposition generated multiwalled carbon nanotubes. J. Phys. Chem. C, 112, 10108-10113.

[11] Guang-yong, Xiong. , Wang, D. Z., \& Ren, Z. F. (2006). Aligned millimeter-long carbon nanotube arrays grown on single crystal magnesia. Carbon , 44, 969-973.

[12] Nii, H., Sumiyama, Y. , \& Kunishige, A. (2008). Influence of diameter on the Raman of multi-walled carbon nanotubes. Applied Physics Express, 1, 064005.

[13] Hata, K. (2004). Water-assisted highly efficient synthesis of impurity-free single-walled carbon nanotubes. Science, 306, 1362-1364.

[14] Iijima, S. (1991). Helical microtubules of graphitic carbon. Nature, 354, 56-58.

[15] Ishikawa, T. (2006). Overview of trends in advanced composite research and applications in Japan. Adv. Compos. Mater., 15, 3-37.

[16] Iwasaki, T., Zhong, G. F., Aikawa, T., Yoshida, T., \& Kawarada, H. (2005). J. Phys. Chem. B, 109, 19556.

[17] Benit, J. M., , J. P. B., \& Lefrant, S. (2002). Low frequency raman studies of muti-wall carbon nanotubes: experiments and theory. Physical review B. Condensed matter and materials physics $073417,66,1-4$.

[18] Jiang, K., Li, Q., \& Fan, S. (2002). Spinning continuous carbon nanotube yarns. Nature, $419,801$.

[19] Hasegawa, K., , S. N., \& Yamaguchi, Y. (2008). Growth window and possible mechanism of millimeter-thick single-walled carbon nanotube forests. Journal of Nanoscience and Nanotechnology, 8, 6123.

[20] Lau, K. T., , C. G., \& Hui, D. (2006). A critical review on nanotube and nanotube/ nanoclay related polymer composite materials. Composites, Part B, 37, 425 .

[21] Zhu, L., Xu, J. , \& Wong, C. P. (2007). The growth of carbon nanotubes stacks in the kinetics-controlled regime. Carbon, 45, 344-348. 
[22] Li, Y., Kinloch, I. A., \& Windle, A. H. (2004). Direct spinning of carbon nanotube fibers from chemical vapor deposition synthesis. Science, 304, 276-278.

[23] Lingbo, Zhu. D. W. H., \& Ching-Ping, Wong. (2006). Monitoring Carbon Nanotube growth by Formation of nanotube stacks and investigation of the diffusion-control kinetics. J.Phys. Chem. B, 110, 5445-5449.

[24] Liu, K., Jiang, K. L., Feng, C., Chen, Z., \& Fan, S. S. (2005). Carbon, 43, 2850.

[25] Matthews, M. J., Pimenta, M. A., \& Endo, M. (1999). Origin of disperive effects of the raman D band in carbon materials. Physical review B, 59, R6585.

[26] Meshot, E. R., \& Hart, A. J. (2008). Abrupt self-termination of vertically aligned carbon nanotube growth. Applied Physics Letters, 92, 113107-113103.

[27] Noda, S., Hasegawa, K., Sugime, H., Kakehi, K., Zhang, Z., Maruyama, S., \& Yamaguchu, Y. (2007). Jpn. J. Appl. Phys, 46, L399.

[28] Oleg, V., \& Yazyev, A. P. (2008). Effect of metal elements in catalytic growth of carbon nanotubes. Physical review letters, 100, 156102.

[29] Li, Qingwen. X. , Xiefei, Z., \& Zhu, Yuntian T. (2006). Sustain growth of ultralong carbon nanotube arrays for fiber spinning. Advanced Materials, 18, 3160-3163.

[30] Brukh, R., \& Mitra, S. (2006). Mechanism of carbon nanotube growth by CVD. Chemical Physics Letters, 424, 126-132.

[31] Xiang, R., , Z. Y., \& Maruyama, S. (2008). Growth deceleration of vertically aligned carbon nanotube arrays: catalyst deactivation or feedstock diffusion controlled? J. Phys. Chem. C, 112, 4892-4896.

[32] Maruyama, S., Einarsson, E., \& Edamura, T. (2005). Growth process of vertically aligned single-walled carbon nanotubes. Chemical Physics Letters, 403, 320-323.

[33] Pal, S. K., , S. T., \& Ajayan, P. M. (2008). Time and temperature dependence of mutiwalled carbon nanotube growth on inconel 600. Nanotechnology , 19, 045610.

[34] Shim, J. S., Yun, Y. H., Cho, W., Shanov, V., Schulz, M. J., \& Ahn, C. H. (2010). SelfAligned Nanogaps on Multilayer Electrodes for Fluidic and Magnetic Assembly of Carbon Nanotubes. Langmuir, 26, 11642-11647.

[35] Stadermann, M., Sherlock, S. P., In, J. B., Fornasiero, F., Park, H. G., Artyukhin, A. B., Wang, Y., De Yoreo, J. J., Grigoropoulos, C. P., Bakajin, O., Chernov, A. A., \& Noy, A. (2009). Mechanism and Kinetics of Growth Termination in Controlled Chemical Vapor Deposition Growth of Multiwall Carbon Nanotube Arrays. Nano Letters, 9, 738-744.

[36] Shanov, V., , W. C., Schulz, M., \& Malik, N. (2008). Advances in synthesis and application of carbon nanotube materials. Materials science and technology, 2253.

[37] Zhao, X., Saito, R., \& Ando, Y. (2002). Characteristic raman spectra of mutiwalled carbon nanotubes. Physica B, 323, 265-266. 
[38] Yun, Y. H., , A. B., Shanov, V. N., \& Schulz, M. J. (2006). A nanotube composite microelectrode for monitoring dopamine levels using cyclic voltammetry and differential pulse voltammetry. 220, $53-60$.

[39] Yun, Y., Shanov, V., Tu, Y., Subramaniam, S., \& Schulz, M. J. (2006). Growth Mechanism of Long Aligned Multiwall Carbon Nanotube Arrays by Water-Assisted Chemical Vapor Deposition. The journal of physical chemistry B, 110, 23920-23925.

[40] Zhong, G., Iwasaki, T., Robertson, J., \& Kawarada, H. (2007). Growth Kinetics of 0.5 cm Vertically Aligned Single-Walled Carbon Nanotubes. J. Phys. Chem. B, 111, 1907 -1910 . 
